\title{
Propagation of nuclear polaritons through a two-target system: Effect of inversion of targets
}

\author{
G. V. Smirnov, ${ }^{1}$ U. van Bürck, ${ }^{2}$ W. Potzel, ${ }^{2}$ P. Schindelmann, ${ }^{2}$ S. L. Popov, ${ }^{1}$ E. Gerdau, ${ }^{3}$ Yu. V. Shvyd'ko, ${ }^{3}$ H. D. Rüter, ${ }^{3}$ and \\ O. Leupold ${ }^{3, *}$ \\ ${ }^{1}$ Russian Research Center “Kurchatov Institute," 123182 Moscow, Russia \\ ${ }^{2}$ Physik-Department, Technische Universität München, 85748 Garching, Germany \\ ${ }^{3}$ Institut für Experimentalphysik, Universität Hamburg, D-22761 Hamburg, Germany
}

(Received 15 October 2004; published 4 February 2005)

\begin{abstract}
The propagation of nuclear polaritons through a scattering system composed of two spatially separated targets was studied. The stainless-steel targets were highly enriched in resonant ${ }^{57} \mathrm{Fe}$ nuclei and had different thicknesses, and, in addition, the resonance width of one target could be increased by cooling it to low temperatures. A nuclear polariton state was created in both targets almost instantly by a short flash of synchrotron radiation. The propagation of the polariton could be perturbed by a time-dependent Doppler shift of the resonance energy in one of the targets. The effect of inversion of the target sequence on the polariton propagation was in the focus of our investigations. It was shown both theoretically and experimentally that in general a two-target scattering system is not commutative.
\end{abstract}

DOI: 10.1103/PhysRevA.71.023804 PACS number(s): 42.50.Fx, 42.50.Gy, 42.25.Bs, 76.80.+y

\section{INTRODUCTION}

\section{A. Nuclear polariton}

A photon traveling in solid matter cannot be treated as an independent entity. The interaction of photons with a crystal produces various kinds of excitations, e.g., phonons (in an ionic crystal), plasmons (in a metal), or excitons (in a semiconductor). Photons and solid-state excitations are dynamically coupled and periodically exchange energy. Thus a mixed state of two quantum-mechanical systems-the electromagnetic field and a solid-state excitation-is created inside the crystal. This state represents a coupled combination of an electromagnetic wave and a medium-polarization wave propagating through the crystal. The quantum of such a mixed state is called a polariton.

In the frequency regions of visible light and microwave radiation there exist several polariton variations tightly related to the character of the induced oscillators: phonon polaritons, plasmon polaritons, and exciton polaritons [1,2]. A very special type of polariton is created when an $\mathrm{x}$ - or $\gamma$-ray photon resonantly interacts with the nuclei in solid matter. Here, far from the optical region, quite different scales of energy (typically $\left.E=10^{4} \mathrm{eV}\right)$, wavelength $(\lambda=1 \AA)$, and time $(t=100 \mathrm{~ns})$ are involved. An ensemble of Mössbauer nuclei in a crystal represents a nuclear medium. There the nuclei act as pure resonant two-level systems. In addition, the radiation sees such a medium as being discrete in space. Therefore, a nuclear ensemble could represent for the radiation a perfect resonating three-dimensional diffraction grating, and spatial interference in resonant scattering by the individual nuclei is expected.

Experiments on nuclear Bragg diffraction, total external nuclear reflection, and nuclear forward scattering (see the review [3]) have proved convincingly that not single nuclei but nuclear ensembles can be involved in the scattering of a

\footnotetext{
*Electronic address: smirnov@polyn.kiae.su
}

single photon. Thus, it was shown that collective nuclear scattering can play a crucial role in the whole picture of interactions of $\mathrm{x}$ - and $\gamma$-ray photons with a nuclear ensemble.

To describe the collective scattering, the concept of a delocalized nuclear excitation similar to the exciton known from optics [4] was introduced in the theory [5-7]. For the interpretation of the collective nuclear response, one may assume the possibility of excitation of each nucleus in accordance with the principle of superposition of states. If the state in which one nucleus is excited and all other nuclei are in the ground state is an elementary state of excitation, then, in this model, the nuclear exciton wave function is a linear superposition of such elementary excitations. Thus the nuclear excitation is delocalized and a photon is shared by many nuclei. This is how the scattering process exhibits a collective character.

The total field in the crystal represents a coherent superposition of the waves "allowed" by the scattering system, i.e., the waves that are solutions of the Maxwell equations of the scattering problem. The waves are dynamically coupled via nuclear currents feeding one another, so that the total field and the excited nuclear polarization wave must be considered as a single entity. To follow the terminology used in optics one should call the entity a "nuclear exciton polariton" or simply a "nuclear polariton."1

In general a nuclear ensemble represents a strongly dispersive, optically active, and diffracting medium. Therefore

\footnotetext{
${ }^{1}$ Presently the term "nuclear exciton" is widely used in the circle of physicists working in the field of nuclear resonant scattering of Mössbauer and synchrotron radiation. This term has its own history. Initially it referred to a delocalized nuclear excited state [5-7] and actually presented a new concept for the description of the coherent scattering of a $\gamma$ quantum by a nuclear ensemble. Later on it was emphasized that a mixed quantum-mechanical state combining the dynamically coupled $\gamma$ quantum and the nuclear ensemble should be considered $[8,20,21]$. The terms "nuclear gamma exciton," "gamma exciton," and "nuclear exciton" were proposed. However, to be in line with the optical community it would be helpful in the
} 
nuclear polaritons can have different polarization states and different propagation vectors. Both propagating and standing wave modes of nuclear polaritons can be realized. In solid matter, a nuclear polariton propagating in the forward direction is always created when the nuclear ensemble is excited by a photon, irrespective of the structure of the nuclear ensemble.

\section{B. Propagation of a nuclear polariton in time}

Synchrotron radiation (SR) sources provide an excellent opportunity to observe the propagation of nuclear polaritons in the forward direction. The energy spectrum of the SR by far exceeds the nuclear resonance range. The excitation of a nuclear target by a SR pulse occurs still within $\sim 0.2 \mathrm{~ns}$, whereas the time response of the nuclei lies in the $100 \mathrm{~ns}$ range due to the sharpness of the resonance. Thus, the primary excitation and the following decay stages are well separated in time, and the nuclear polariton propagation through the target is easily traced.

The most prominent features of the propagation of the nuclear polariton are the dynamical and the quantum beats (DB's and QB's) of the forward scattered intensity (see, e.g., the reviews $[8,9])$. In the energy space both kinds of beats can be understood as the result of interference of the forward scattered spectral components of the radiation. The QB arises in the presence of hyperfine splitting of the nuclear levels and is due to inter-resonance interference of the radiation components. The DB (also known as the propagation beat in optics) arises due to the intraresonance interference of the radiation components. It is especially representative for thick nuclear targets where the spectrum of the forward scattered radiation acquires a double-hump structure.

On the time scale, the DB reveals the dynamics of the polariton propagation, namely, the dynamics of the energy exchange between the electromagnetic field and the nuclear excitation: sequential processes of absorption and reemission of radiation by the nuclei (see Fig. 7 in [8]). These processes are regulated by the coupling of the radiation to the nuclear currents of the ensemble. The radiative coupling is also responsible for the enlarged decay rate at the initial stage of the polariton propagation $[8,9]$.

In the forward scattering geometry the coherent coupling of radiation field and nuclear oscillators is established and maintained over the whole scattering ensemble along the beam even if the ensemble is split into several spatially separated targets. In the process of propagation the coherent field emitted by a nuclear layer is traveling in the forward direction. Therefore the field reemitted by an upstream layer can be reabsorbed only by a downstream layer. Likewise, in the case of two spatially separated targets the field leaving the upstream target drives the nuclear oscillators in the downstream target. Splitting a target into parts allows one to easily

future to use the term "polariton" which is well established in this field for the description of similar optical phenomena (earlier this term was used in [22]). The term "nuclear exciton" we will preserve for the description of nuclear polarization, i.e., in its original meaning. modify the radiative coupling between the targets by manipulating its parts, and, in this way, to manipulate the propagation of a nuclear polariton through the system.

The effect of radiative coupling between two separated targets was thoroughly investigated in nuclear forward scattering of synchrotron radiation [10,11]. Stunning effects have in particular been observed in a target system composed of two targets $N$ (narrow) and $W$ (wide) having highly different nuclear resonance linewidths of about 1 and 10 natural linewidths $\Gamma_{0}$, respectively [10]. In particular, after an initial decay with strongly enlarged decay rate, the reemission in the forward direction did not recover at later times but remained almost zero. Moreover, this behavior was found to be independent of the target sequence, that is, when the targets playing the role of driver and oscillator with very different decay parameters were inverted.

In order to analyze these surprising findings and to gain deeper insight into the physics of the propagation of nuclear polaritons through a system of two targets a method of manipulating the radiative coupling of the targets developed in [11] was employed in the present study. In this method, the radiative coupling of the two targets can be instantly interrupted by means of a stepwise Doppler energy shift applied to one of the targets. Effects caused by the inversion of the targets under these conditions were the focus of the present study.

The paper is organized as follows. In Sec. II we present the theory describing the propagation of a nuclear polariton through a system of two targets in the presence of an external perturbation of the system. Section III is devoted to the description of the experiments and of their results. It contains also the analysis of the experimental results. A summary and conclusions are given in Sec. IV.

\section{PROPAGATION OF A NUCLEAR POLARITON THROUGH A SYSTEM OF TWO TARGETS: THEORY}

The propagation of a polariton is reflected in the temporal response of the scattering system in the forward direction. Let the incident radiation be represented by a $\delta$ function $\delta(t)$. Then the delayed response of a single resonance nuclear target at time $t$ is described by the following wave packet [12]:

$$
W(t, T)=\text { const } \times \frac{1}{2 t_{0}} T e^{i \omega_{0} t-q t /\left(2 t_{0}\right)} \frac{J_{1}\left(\sqrt{T t / t_{0}}\right)}{\sqrt{T t / t_{0}}}
$$

where $T=n \sigma_{0} f$ is the Mössbauer thickness of the target, with $n$ being the number of resonant nuclei per unit area, $\sigma_{0}$ the maximal resonance cross section of the nuclear transition, and $f$ the Lamb-Mössbauer factor; $\omega_{0}$ is the resonance frequency, $t_{0}=\hbar / \Gamma_{0}$ is the lifetime of the nuclear excited state with natural width $\Gamma_{0}$, and $J_{1}$ is the Bessel function of the first kind and first order. The parameter $q$ takes a Lorentzian broadening of the resonance into account (see [8]).

$W(t, T)$ is the probability amplitude for a $\gamma$ quantum to be coherently emitted at time $t$ into the forward direction by a nuclear target of thickness $T$. This amplitude is directly related to the electromagnetic wave field behind the target. Any delayed $\gamma$ quantum behind the target, however, is a proof of 

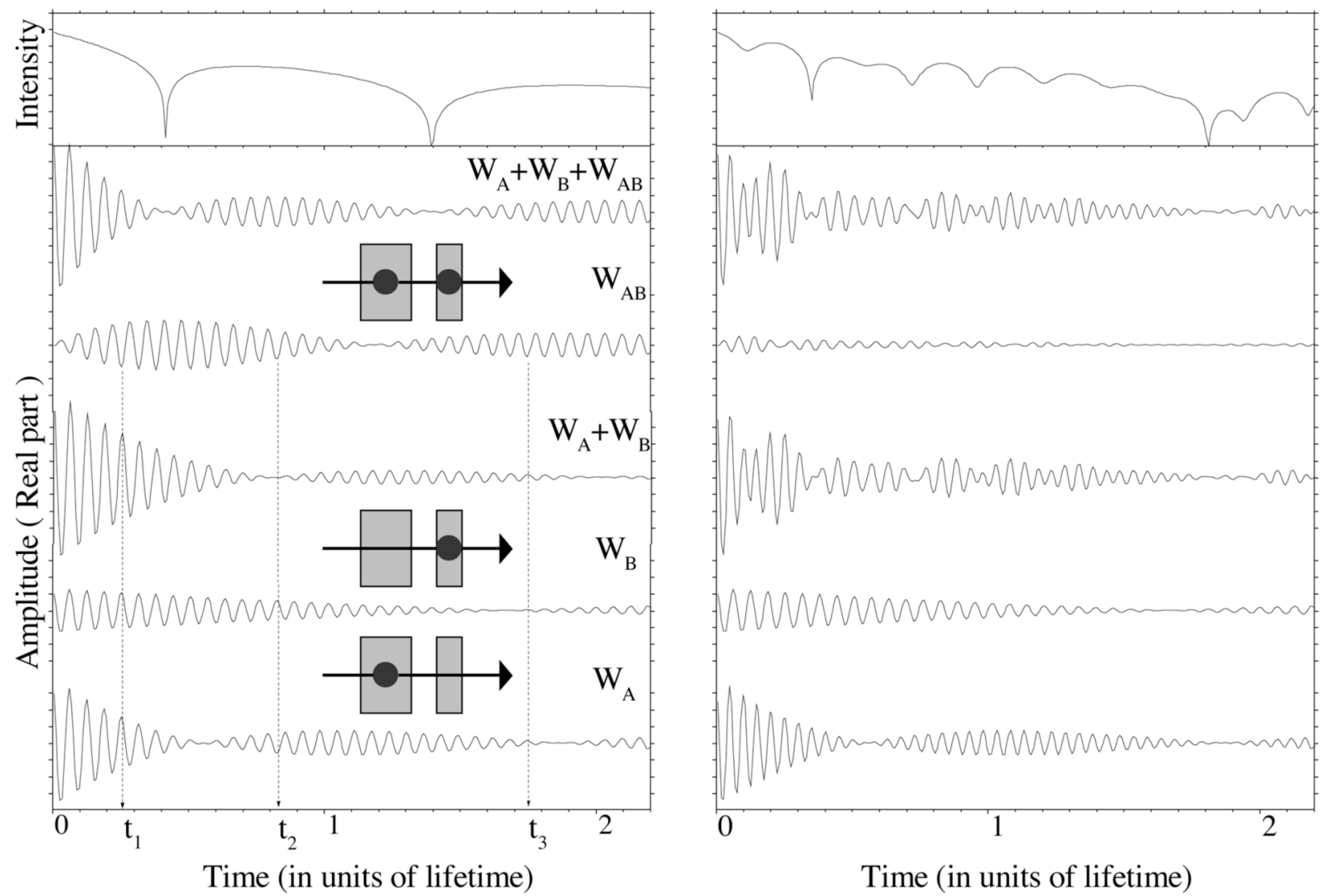

FIG. 1. Evolution of nuclear forward scattering of synchrotron radiation from a system composed of two targets exhibiting each a single line resonance and having effective thicknesses $T_{A}=27$ and $T_{B}=9$. The targets are in resonance at the left-hand side, whereas at the right-hand side the resonance lines are separated by an energy shift of $\sim 50 \Gamma_{0}$. The partial scattering paths are displayed in the insets where dark circles indicate the interaction of the radiation with the nuclei in the targets. The three paths are described by the probability waves $W_{A}, W_{B}$, and $W_{A B}$, respectively. The summary wave and intensity are shown in the two uppermost panels. Amplitudes are always given in linear scale, intensities in logarithmic scale.

previous creation of a nuclear polariton and its propagation through the target. One should keep in mind that $W(t, T)$ describes only the electromagnetic wave (in free space behind the target), whereas the polariton is composed of both an electromagnetic wave and a nuclear polarization wave inside the target. The exchange of energy between these two subsystems is described by the Bessel modulation in Eq. (1) (see Fig. 7 in Ref. [8]).

Thus the modulation of the wave packet $W(t, T)$ represents the multiple scattering of radiation by the nuclei which occurs during the polariton propagation through the target [8]. The wave packet exhibits a node-antinode structure. The antinodes in the wave packet reveal sequential nuclear scattering stages. Each nuclear resonant scattering results in a phase shift of $-\pi$ of the wave where the fraction $-\pi / 2$ originates from the scattering at resonance and another fraction of $-\pi / 2$ is due to forward scattering of a plane wave by a plane scatterer (Fresnel interference).

We now consider a system composed of two targets $A$ and $B$ characterized by effective thicknesses $T_{A}$ and $T_{B}$, resonance frequencies $\omega_{A}$ and $\omega_{B}$, and resonance broadening parameters $q_{A}$ and $q_{B}$, respectively. We assume the target $A$ to be the upstream and $B$ the downstream part of the composed system. As shown in [8], the wave packet representing the delayed coherent response of the system of two separated targets $A$ and $B$ is a composition of three contributions,

$$
W_{A+B}(t)=\left\{W_{A}(t)+W_{B}(t)+W_{A B}(t)\right\}
$$

with

$$
W_{A B}(t)=-\int_{0}^{t} d t^{\prime} W_{B}\left(t-t^{\prime}\right) W_{A}\left(t^{\prime}\right)
$$

Each of the constituent wave packets $W_{A}(t), W_{B}(t)$, and $W_{A B}(t)$ represents one of three scattering paths. These are nuclear scattering of SR by target $A$ or $B$, as if each target were alone in the beam, and scattering of SR by target $A$ and thereafter scattering of the delayed nuclear radiation from target $A$ by $\operatorname{target} B$, i.e., the sequential scattering of radiation by the upstream and downstream parts of the scattering system. Schematically the scattering paths are shown in the insets of Fig. 1 where the dark circles symbolize the events of nuclear excitation inside the targets for each scattering path. Correspondingly, the wave packets $W_{A}$ and $W_{B}$ represent the delayed responses of the relevant targets [those given by Eq. 
(1)], whereas the wave packet $W_{A B}$ is their convolution. It is found by using the response function technique.

The wave packet $W_{A B}$ represents the third scattering path and reflects the resonant interaction of radiation emerging from the upstream target with the nuclei of the downstream target. Thus in accord with its nature this wave packet represents the radiative coupling of the nuclear ensemble in target $A$ with that in target $B$. In the integral in Eq. (2), the running time $t^{\prime}$ is the emission time from target $A$ as well as the excitation time of target $B$; the time $t$ is the emission time from target $B$ as well as the observation time (the time of flight of radiation between the targets and between the second target and the detector does not count and is omitted). Time zero is set by the arrival of the SR pulse at the detector. In brief, in the third scattering path the polariton propagates in target $A$ within the time interval from 0 to $t^{\prime}$ and in target $B$ from $t^{\prime}$ to $t$.

The propagation time of a nuclear polariton can vary in broad limits. The more time the polariton was propagating through the system the more collisions of the $\gamma$ quantum with the nuclear ensemble occurred. The scale of the propagation time can be attached to the lifetime of the excited nuclear state.

Thus the overall delayed response of the system is described as the interference of the three probability waves $W_{A}(t), W_{B}(t)$, and $W_{A B}(t)$. As an illustration we consider a hypothetical nuclear $\gamma$ resonance in the absence of hyperfine splitting. Let the scattering system be composed of two targets having identical characteristics except for their thicknesses. The probability waves (in fact the real amplitudes) of the particular scattering channels are displayed in Fig. 1 for targets having thicknesses $T_{A}=27$ and $T_{B}=9$. In order to make the phase relations of the interfering waves visible their carrier oscillations are artificially slowed down in the figure.

At the left-hand side of the figure the case is illustrated where the targets have the same resonance frequency. The pronounced node-antinode structure of the summary wave $\left\{W_{A}+W_{B}+W_{A B}\right\}$ is a dramatic consequence of the propagation of the nuclear polariton through the targets. As discussed in [8] (Sec. 6.3), the space-time modulation of the propagating field and the nuclear excitation amplitudes in the target reveal sequential events of absorption and reemission of radiation by the nuclei. The number of antinodes in the transmitted field shows the number of scattering stages which occur within the observation time window inside the given target. For instance, regarding the emission from target $B$ alone, two scattering stages are revealed within $B$, whereas for the combined target, three scattering stages are traced, see the structure of the relevant wave packets at the left-hand side of Fig. 1.

While the wave packets $W_{A}$ and $W_{B}$ correspond to free emission from the targets $A$ and $B$ after the prompt excitation of the targets by the SR pulse, the wave packet $W_{A B}$ describes the emission from the nuclei in $\operatorname{target} B$ when they are continuously driven by the radiation incoming from target $A$. Obviously, this radiative coupling of the targets is most influential when the radiation is in resonance with the nuclei, i.e., when $\omega_{A}=\omega_{B}$ : note the well developed probability wave $W_{A B}$ at the left-hand side of the figure. In the oppo- site case when $\left|\omega_{A}-\omega_{B}\right| \gg \Gamma_{0}$, illustrated in the right-hand set of panels of Fig. 1, the radiative coupling is of minor importance: note the weak probability wave $W_{A B}$. It should also be strongly decreased when the widths of the resonances in the up- and downstream targets are essentially different. From the point of view of radiative coupling the considered extreme cases are characterized as the coupled and decoupled states of the system. In the decoupled state mainly the interference of the two scattering channels described by the $W_{A}$ and $W_{B}$ waves determines the whole scattering picture. For instance, in the case of shifted resonances their interference yields a prominent quantum beat of the scattering intensity (the uppermost right-hand panel), which occurs at the difference frequency $\Delta \omega=\omega_{A}-\omega_{B}$ [9]. In our example it corresponds to an energy shift $\hbar \Delta \omega=50 \Gamma_{0}$. However, we emphasize that in spite of such a large separation of the resonances the radiative coupling is still present, although with drastically reduced amplitude; compare the amplitudes of the wave packets $W_{A B}$ in Fig. 1 (at the left- and right-hand sides).

We consider now the interference of the wave packets representing the three scattering paths when the targets are radiatively coupled. Regarding interference one has to take into account both the amplitude and the phase relationship of the wave packets. Several characteristic time moments are marked in Fig. 1 (left-hand side). At the first scattering stage, e.g., at time $t_{1}$, the waves $W_{A}$ and $W_{B}$ having the same carrier frequency are in phase. At the same time they are in opposite phase to the wave packet $W_{A B}$. The latter presents the nuclear coherent scattering of the $W_{A}$ wave by $\operatorname{target} B$. Because of the additional event of nuclear coherent scattering the phase of the $W_{A B}$ packet is shifted by $-\pi$. For the same reason the phase of the $W_{A}$ wave at time $t_{2}$ is shifted by $-\pi$ with respect to the phase of the $W_{B}$ wave because $t_{2}$ belongs already to the second scattering stage of target $A$. At the time $t_{2}$ all three scattering paths contribute to the resultant wave with about the same amplitude whereas at time $t_{3}$ the resultant wave is mainly built by scattering through the radiative coupling path, i.e., by the wave $W_{A B}$.

Equation (2) describes the coherent response of the composite target when its parts are at rest. In the presence of a relative motion between the targets Eq. (2) has to be modified. We let target $A$ move with respect to $\operatorname{target} B$ at an arbitrary velocity $v(t)$ along the beam direction. Because of this motion the wave packet emitted by target $A$ acquires an additional time-dependent phase

$$
\varphi(t)=\frac{2 \pi}{\lambda} \int_{0}^{t} d t^{\prime} v\left(t^{\prime}\right)
$$

where the integral represents the displacement of target $A$ during the time $t$. The delayed response of the system is then as follows:

$$
W_{A+B}(t)=e^{i \varphi(t)} W_{A}(t)+W_{B}(t)-\int_{0}^{t} d t^{\prime} W_{B}\left(t-t^{\prime}\right) e^{i \varphi\left(t^{\prime}\right)} W_{A}\left(t^{\prime}\right) .
$$

Any relative motion between the targets leads to a perturbation of the nuclear polariton propagation through the system. 
It is revealed (a) in the phase modulation of the packet $W_{A}$ and (b) in the time-dependent variation of the radiative coupling term $W_{A B}$. Different cases of the dynamic perturbation of the polariton propagation have already been studied. They were determined by the character of the motion: e.g., a sinusoidal displacement of the target was investigated in [13-15], and a trapezoidal displacement in [11]. In the last paper the transitions between coupling and decoupling regimes during the polariton propagation were investigated thoroughly. In these studies the question was raised whether the propagation of a nuclear polariton through the system is invariant to the inversion of the targets. It is obvious that the response of the system is invariant to inversion if the constituent targets are identical, i.e., $W_{A} \equiv W_{B}$. We now find the response of a system in which the sequence of nonidentical targets is inverted. We consider the scattering system where $B$ is the upstream and $A$ the downstream target. As in the initial case $\operatorname{target} A$ is moved. The delayed response of the inverted system is found as

$W_{B+A}(t)=W_{B}(t)+e^{i \varphi(t)} W_{A}(t)-\int_{0}^{t} d t^{\prime} W_{A}\left(t-t^{\prime}\right) e^{i \varphi\left(t, t^{\prime}\right)} W_{B}\left(t^{\prime}\right)$.

With respect to target $B$ the moved $\operatorname{target} A$ is now in the position of the responding target. Its excitation time is $t^{\prime}$. Therefore the phase factor due to the motion in this case is a function of the times $t^{\prime}$ and $t$. Hence, the phase is given by the integral $\varphi\left(t, t^{\prime}\right)=(2 \pi / \lambda) \int_{t^{\prime}}^{t} d t^{\prime \prime} v\left(t^{\prime \prime}\right)$, i.e., $\varphi\left(t, t^{\prime}\right)=\varphi(t)$ $-\varphi\left(t^{\prime}\right)$. The expression for the response of the inverted system now reads

$$
\begin{aligned}
W_{B+A}(t)= & W_{B}(t)+e^{i \varphi(t)} W_{A}(t) \\
& -e^{i \varphi(t)} \int_{0}^{t} d t^{\prime} W_{A}\left(t-t^{\prime}\right) e^{-i \varphi\left(t^{\prime}\right)} W_{B}\left(t^{\prime}\right) .
\end{aligned}
$$

Comparing Eqs. (4) and (6) it is seen that only the partial wave describing the radiative coupling of the targets is affected by the inversion. To reveal the difference between the wave packets $W_{A B}$ and $W_{B A}$ we transform $W_{B A}$ by first introducing the new variable of integration $\tilde{t}=t-t^{\prime}$ and then by naming the variable $\tilde{t}$ as $t^{\prime}$ in the new integral (both times run over the same time interval). After such a procedure we can directly compare the wave packets $W_{A B}$ and $W_{B A}$ :

$$
\begin{gathered}
W_{A B}(t)=-\int_{0}^{t} d t^{\prime} W_{B}\left(t-t^{\prime}\right) e^{i \varphi\left(t^{\prime}\right)} W_{A}\left(t^{\prime}\right), \\
W_{B A}(t)=-\int_{0}^{t} d t^{\prime} W_{B}\left(t-t^{\prime}\right) e^{i \varphi(t)} e^{-i \varphi\left(t-t^{\prime}\right)} W_{A}\left(t^{\prime}\right) .
\end{gathered}
$$

As seen, the convoluted packets are now identical but their mutual phasing is different. We have to compare the phases $\varphi\left(t^{\prime}\right)$ and $\varphi(t)-\varphi\left(t-t^{\prime}\right)$. The phase here represents the length of the additional optical path arising from the displacement of the moved target; see Eq. (3). In the given case one has to compare the displacements at different stages of motion but during equal time intervals: for the $A B$ sequence of the tar-

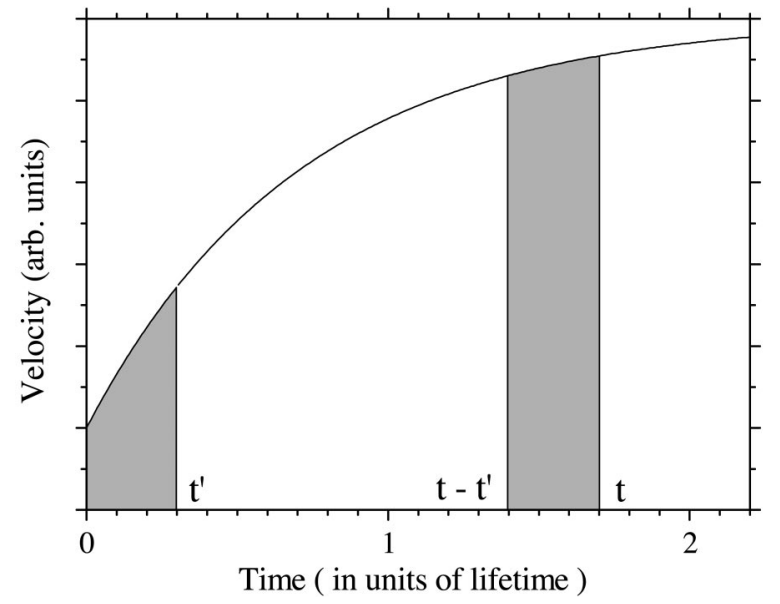

FIG. 2. Comparison of the relative displacements of the targets (dashed areas) during equal time intervals but different stages of motion. The displacements determine the relative phasing between exciting field and target response in the third scattering path in the cases of direct and inverse sequences of the targets; see Eqs. (7) and (8).

gets from 0 to $t^{\prime}$ and for the sequence $B A$ from $t-t^{\prime}$ to $t$. These displacements are shown in Fig. 2 as marked areas. Only for a motion with constant velocity (including zero velocity) are these displacements identical at any observation time $t$. And only for this type of motion is the response of the scattering system invariant to the change of the sequence of the targets, even if the targets have different thicknesses or (and) different resonance widths. If a motion with a constant velocity is applied the separation of the resonant energies of the targets remains constant during the scattering process. Thus, more generally speaking, inversion does not matter when the relative positions of the target resonances do not change while the polariton propagates through the system. However, when a time-dependent shift between the resonance energies occurs, the time response of the scattering system is different for the different sequences of the targets.

The effect of inversion for the same set of targets is illustrated in Fig. 3. Target $A$ is assumed to be moved with constant acceleration according to the law $v(t)=a\left(t-t_{e} / 2\right)$, where $a=$ const stands for the acceleration and $t_{e}$ is the end of the time window. The insets at the left- and right-hand sides of the figure show the sequences of the targets. Obviously, the strongest effect of inversion can be expected when the radiative coupling of the targets is still influential, i.e., when the separation of the resonances in the targets is changed only in the limits of a few resonance widths. Thus in our example an acceleration is taken to yield a separation of the resonances in the targets in the limits of $\pm 7.5 \Gamma_{0}$. Comparing the temporal responses of the system (shown in the uppermost panels of Fig. 3) we can see a clear effect due to the inversion of the targets. The difference in the responses originates from the completely different radiative coupling of the targets. When the polariton propagation is perturbed, inversion of the targets yields a strong amplitude and phase modulation of the relevant wave packet; compare the $W_{A B}$ and $W_{B A}$ packets in the upper part of the figure. For reference, the $W_{A B}$ and $W_{B A}$ packets are also shown for the case 

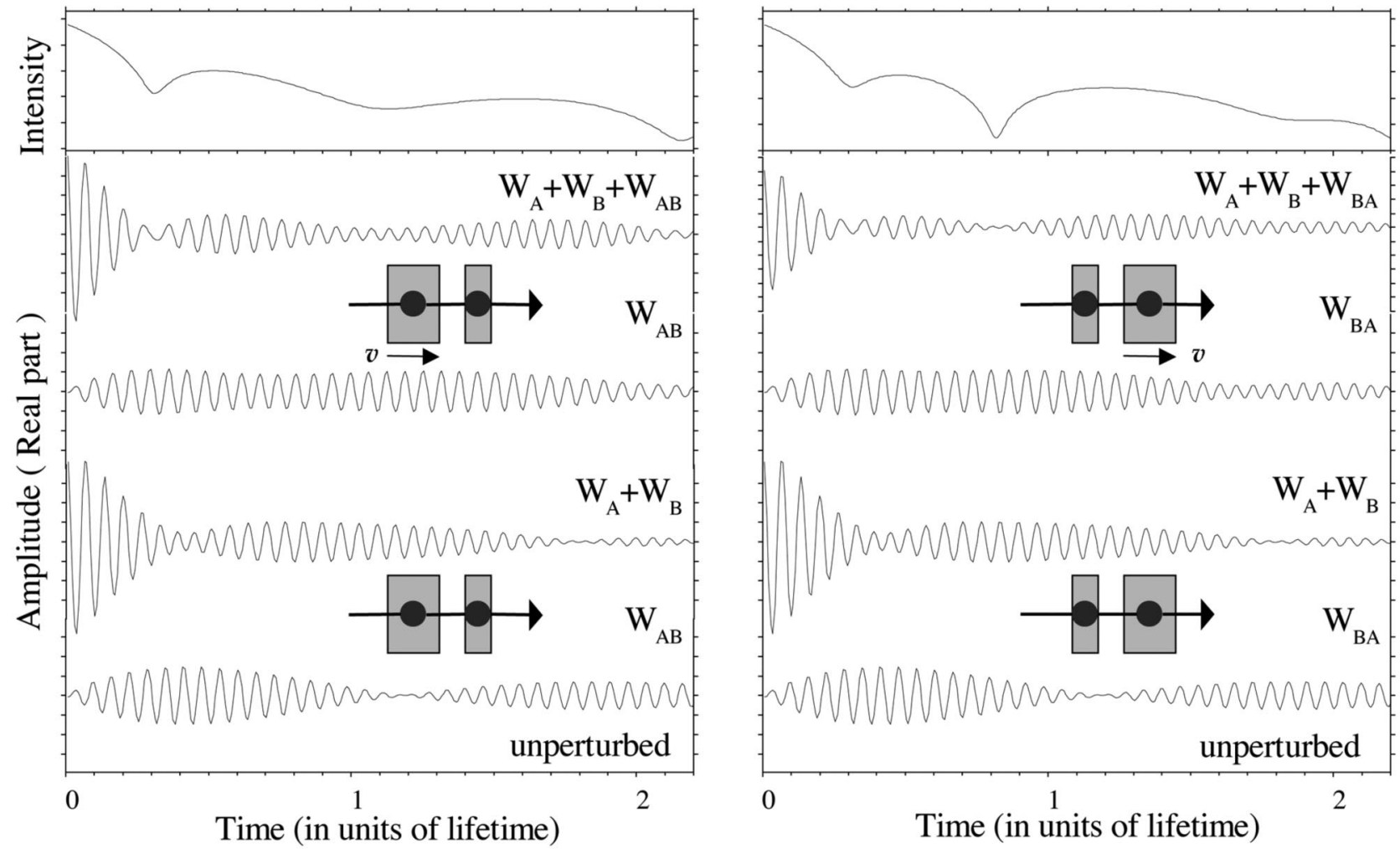

FIG. 3. Evolution of nuclear forward scattering of synchrotron radiation from a system composed of two targets exhibiting single line resonances and having effective thicknesses $T_{A}=27$ and $T_{B}=9$. Target $A$ is moved with constant acceleration (see the upper insets and text). The sequence of the targets is different at the left- and the right-hand sides: $A B$ and $B A$, respectively. The third scattering path is modified due to inversion; compare the corresponding probability waves $W_{A B}$ and $W_{B A}$ in the middle panels. At the same time, inversion does not result in a modification of this path in the absence of a perturbation; compare the same waves in the bottom panels. The effect of inversion in the perturbed system is well seen in the shape of the summary wave and of the intensity shown in the uppermost panels.

that there is no perturbation of the polariton propagation (the panels at the bottom of the figure). An inversion effect is absent here.

Concerning the interference of the waves of the other two scattering paths, it is not sensitive to inversion (as was mentioned above); compare the summary wave packets $\left\{W_{A}\right.$ $\left.+W_{B}\right\}$ at the left- and right-hand sides. As seen from the figure, the amplitudes of $W_{A B}$ and $\left\{W_{A}+W_{B}\right\}$ (left-hand side) and of $W_{B A}$ and $\left\{W_{A}+W_{B}\right\}$ (right-hand side) are comparable for most of the time. That is why a modification of the radiative coupling is so influential in the summary waves $\left\{W_{A}+W_{B}+W_{A B}\left(W_{B A}\right)\right\}$.

Finally, one can formulate a general rule concerning the effect of inversion. The propagation is invariant to the inversion of the targets if the separation of the resonance energies in the targets is not changed while the polariton propagates through the system. Otherwise the polariton propagation is sensitive to the target inversion.

\section{PROPAGATION OF A NUCLEAR POLARITON THROUGH A SYSTEM OF TWO TARGETS: EXPERIMENT}

The measurements were carried out at HASYLAB (Hamburg) at the wiggler beamline BW4. Two kinds of scattering systems were investigated. The first one consisted of two targets identical in all nuclear resonance parameters except for their effective resonance thickness $T$. The second scattering system was composed of two targets having, in addition to different thickness parameters, different nuclear resonance widths. Both targets were prepared of stainless-steel $\mathrm{Fe}_{55} \mathrm{Cr}_{25} \mathrm{Ni}_{20}$ enriched up to $95 \%$ in ${ }^{57} \mathrm{Fe}$. The targets were fabricated from foils having thicknesses of $\sim 1.2, \sim 2.3$, and $\sim 2.5 \mu \mathrm{m}$.

Nuclear polaritons with an energy of $14.4 \mathrm{keV}$ were created by SR flashes in the scattering system. The lifetime of the employed nuclear state in ${ }^{57} \mathrm{Fe}$ is $\sim 141 \mathrm{~ns}$. The propagation of nuclear polaritons was studied under conditions where the radiative coupling of the targets was temporarily perturbed. Such perturbations were created by a sudden motion of one of the targets while the polariton was propagating through the system. In all experiments the moved target was a foil of $1.2 \mu \mathrm{m}$ in thickness and $5 \mathrm{~mm}$ in diameter. Further on we call it target $A$. It was glued to a driver which was manufactured of a piezoelectric polymer foil, polyvinylidene fluoride (PVDF) covered by a conducting layer of nickelaluminum to which a voltage signal was applied. This voltage signal produced the motion of target $A$. Its velocity can be described by rising and falling exponentials, as expected with our experimental setup, where the covered PVDF acts as a capacitor. For further details see Ref. [11]. The velocity 


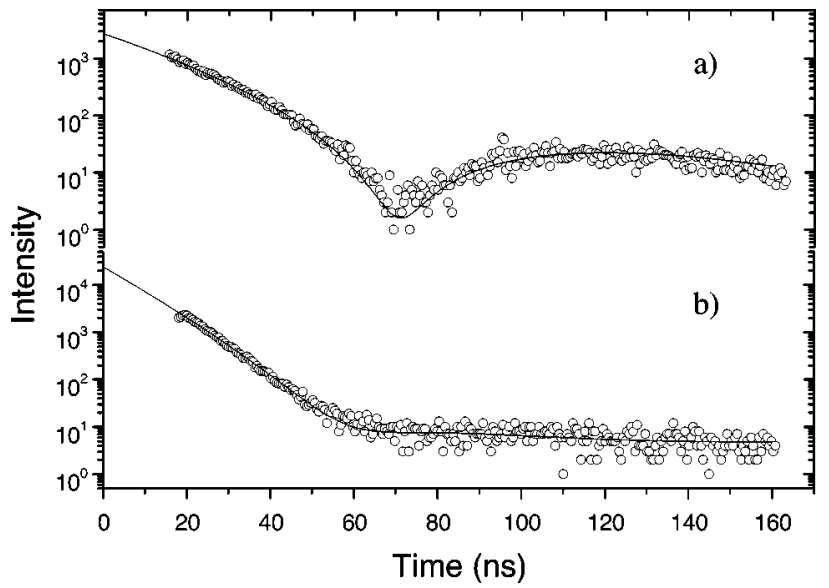

FIG. 4. Experimental time dependences of nuclear forward scattering of synchrotron radiation from a system of two targets $A$ and $B$ manufactured of stainless-steel foils (a) having thicknesses $\sim 1.2$ and $\sim 2.3 \mu \mathrm{m}$, respectively, and being kept at room temperature; (b) having thicknesses $\sim 1.2$ and $\sim 2.5 \mu \mathrm{m}$, respectively, but with target $B$ being cooled to $4.2 \mathrm{~K}$. Due to cooling the resonance line in target $B$ is broadened to $\sim 10 \Gamma_{0}$. Solid lines are computer simulations based on Eqs. (1) and (2).

profile is displayed in the bottom panels of Figs. 6, 7, 9, and 10.

The time of perturbation of the nuclear polariton was synchronized with the arrival of the SR pulse and could be shifted with respect to this arrival time (see Ref. [11] for details).

\section{A. Inversion of targets of different thicknesses}

In these measurements two targets of $\sim 1.2$ and $\sim 2.3 \mu \mathrm{m}$ thickness were used as parts $A$ and $B$ of the scattering system, respectively. Both targets were kept at room temperature where they exhibited almost natural nuclear resonance width, not taking into account the thickness broadening. The polariton propagation in the observation window is characterized by single- and double-scattering stages. This is well seen in Fig. 4(a) where the forward scattering of the SR pulse by the system of two targets is shown in the absence of perturbation. The first scattering stage lies within the time interval 0-70 ns whereas the second stage occupies all of the remaining observation window. The observed intensity evolution does not depend on the target sequence in this case.

To reveal the polariton propagation through the system the time dependences of nuclear forward scattering of synchrotron radiation pulses were measured for the direct $A B$ and inverted $B A$ sequences of the targets. A series of such dependences for different starting times $t_{01}$ of the perturbation of the polariton with respect to the SR flash was obtained. Figure 5 displays a set of selected dependences where the perturbation started within a wide time range from 17 to $120 \mathrm{~ns}$. The left- and the right-hand panels of the figure refer to the direct and inverted orders of the targets, respectively.

The propagation of the nuclear polariton is drastically affected by the perturbation. First of all, the perturbation causes beats of the transmitted intensity which coincide with the time window of the perturbation. The frequency and shape of the beats are determined by the velocity profile as discussed in detail in [11]. In addition to the beats a longlasting influence of the perturbation on the overall shape of the transmitted intensity is observed. For instance, it is well seen that the perturbation occurring at early times essentially extends the first scattering stage until about 100 ns (see bottom panels).

As to the beats, they change their initial phase while the perturbation is shifted from the first to the second scattering stage; compare the dependences referring to the starting times of 44 and $82 \mathrm{~ns}$. Starting at $44 \mathrm{~ns}$ the perturbation causes initially a destructive interference effect, whereas starting at $82 \mathrm{~ns}$ it results in a constructive interference pattern, which is especially pronounced for the direct sequence of targets (left-hand panel). Similar changes of the beat structures were observed in [11].

From the general behavior of the scattering patterns it is also seen that the perturbed system is not commutative. This noncommutativity is most clearly observed when the perturbation occurs within the second scattering stage, i.e., starting at $82 \mathrm{~ns}$ and later. Upon perturbation starting at times 111 and $120 \mathrm{~ns}$, beating of the scattering intensity proceeds even in the opposite phase for the direct and inverted sequences of the targets.

We now shall analyze in detail the effects of perturbation and of inversion for the extreme cases, where the perturbation occurs at the late and at the early stages of scattering.

\section{Late perturbation}

In Fig. 6 the scattering picture is presented where the perturbation started $\sim 120$ ns after the creation of the polariton. The perturbation is caused by a short motion of target $A$ for about $50 \mathrm{~ns}$. The velocity of the motion is displayed in the bottom panels of the figure. The constituent wave packets are presented in the figure again as the real parts of wave amplitudes. The nature of the wave packets was discussed in the previous section. The wave $W_{A}$ reveals that target $A$ stays in the single-scattering stage in the entire observation window. The carrier frequency of the wave is modulated due to the perturbation within the time interval 120-160 ns. The wave $W_{B}$ reveals both first and second scattering of the radiation in $\operatorname{target} B$ within the observation windows 0-100 and 100-160 ns, respectively. Both waves stay unchanged after the inversion of the targets. The waves $W_{A B}$ and $W_{B A}$ represent the radiative coupling of the downstream target with the upstream target for the direct and inverted sequences of targets, respectively. Only these waves are sensitive to inversion. Since in the considered case the targets are in resonance most of the time from $t=0$ to $120 \mathrm{~ns}$, the radiative coupling can result in the excitation of the nuclei in the downstream target to their maximum amplitude. The coupling is perturbed only at the end of the time window. It is well seen that the effect of perturbation is different for the different sequences of the targets: the $W_{A B}\left(W_{B A}\right)$ waves are modified differently in amplitude and phase during the time interval 120-160 ns. The average velocity of the motion corresponds to a shift of the resonances in the targets by about $70 \Gamma_{0}$. So one can expect a complete interruption of the ra- 

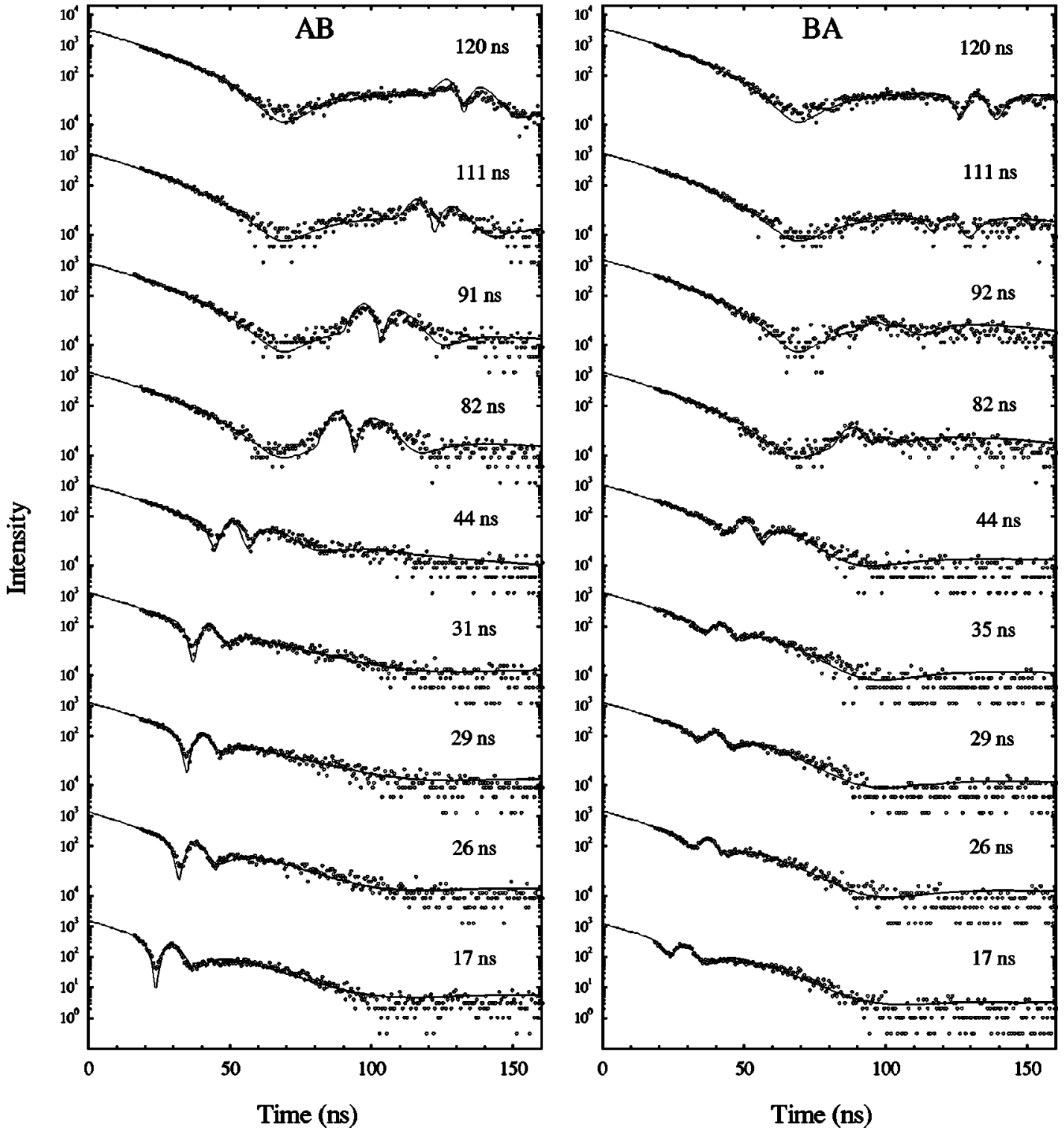

FIG. 5. Experimental time dependences of nuclear forward scattering of synchrotron radiation from a system of two targets $A$ and $B$ manufactured of stainless-steel foils having thicknesses $\sim 1.2 \mu \mathrm{m}\left(T_{A} \approx 10.6\right)$ and $\sim 2.3 \mu \mathrm{m}\left(T_{B} \approx 20.2\right)$, respectively. Both targets are kept at room temperature. The scattering is perturbed by a sudden motion of target $A$ within $\sim 50 \mathrm{~ns}$. The motion starts at different times $t_{01}$ from 17 to $120 \mathrm{~ns}$, as indicated, perturbing the scattering in characteristic stages of its development. The results of the measurements for the direct $A B$ and inverse $B A$ sequences of the targets are presented at the left- and right-hand sides, respectively. Solid lines are computer simulations based on Eqs. (4) and (6).

diative coupling between the targets from time $120 \mathrm{~ns}$ on. After this time the excitation produced in the downstream targets via the third scattering channel should exhibit mainly a free decay. For the sequence $A B$ the deexcitation of the downstream thick target $B$ should be coherently enlarged as compared to the deexcitation of the downstream thin target $A$ in the sequence $B A$. That is why the probability wave $W_{A B}$ decays faster than $W_{B A}$.

In the summary wave $\left\{W_{A}+W_{B}+W_{A B}\left(W_{B A}\right)\right\}$ a pronounced amplitude modulation is seen during the perturbations which results in the beats of the scattering intensity. However, the beats start with opposite phases for the different sequences of the targets.
The primary reason for this noncommutativity of the scattering system is the different thickness of the targets. For the direct $A B$ sequence of the targets the waves $W_{A B}$ and $W_{B}$ oscillate in phase for most of the time after $\sim 100 \mathrm{~ns}$ because after this time they both are generated by double-nuclearresonant scattering. Just at the begin of the perturbation at $120 \mathrm{~ns}$ both waves are in opposite phase with respect to the $W_{A}$ wave. That is why the beating starts with constructive interference of the $\left\{W_{B}+W_{A B}\right\}$ and $W_{A}$ waves when the phase of the latter is modulated.

However, in the case where the targets are inverted the waves $W_{B A}$ and $W_{A}$ oscillate in opposite phase because they are created by double- and single-nuclear scattering, respec- 

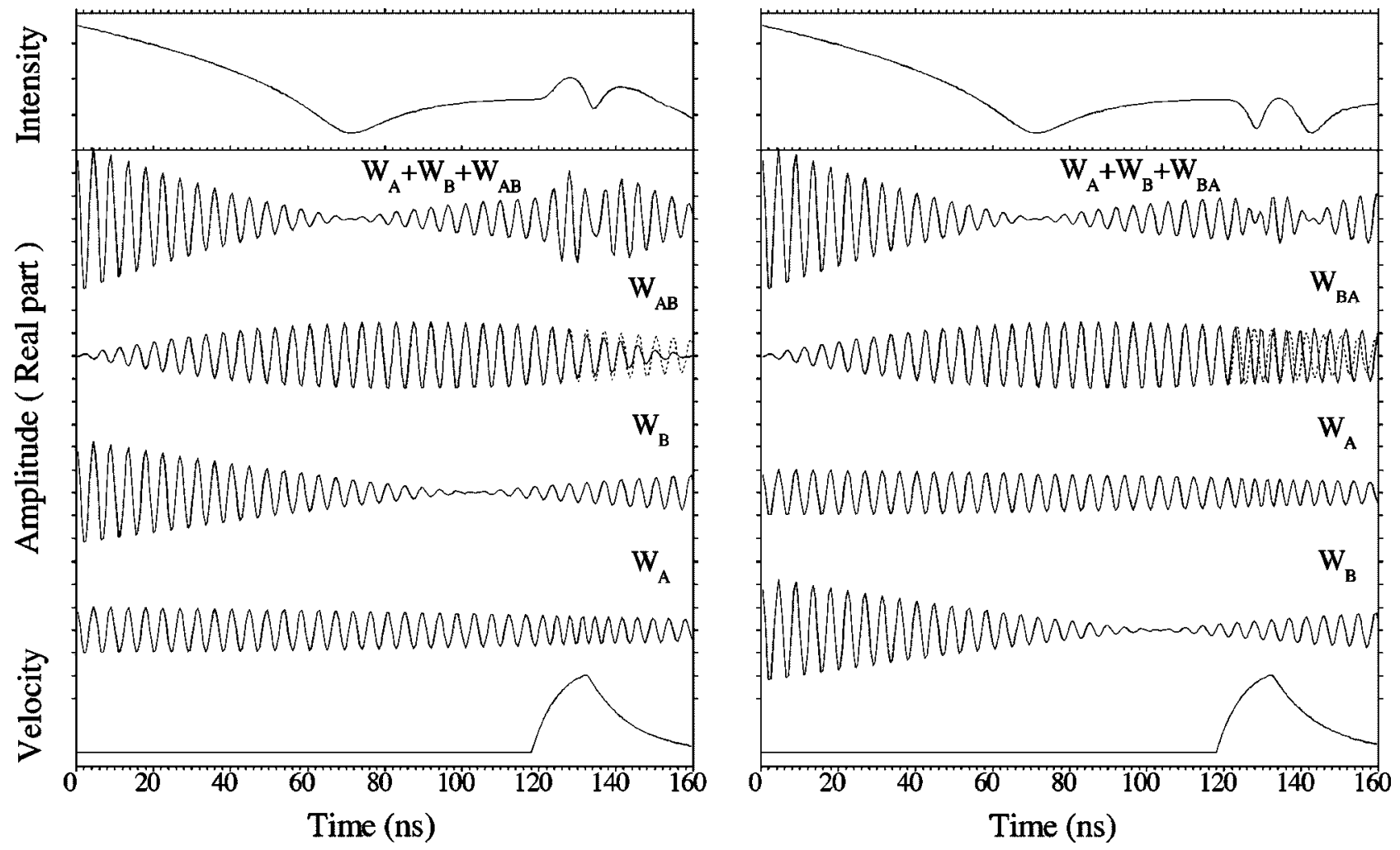

FIG. 6. Effect of inversion at late perturbation (see Fig. 5). The left (right) panel corresponds to the direct (inverted) target sequence $A B(B A)$, respectively. The velocity of $\operatorname{target} A$ is depicted by the solid line in the bottom panel. With the inverted sequence of the targets the quantum beats caused by the perturbation proceed with opposite phases (see text). The probability waves $W_{A B}$ and $W_{B A}$ in the absence of perturbation are displayed by dashed lines.

tively. Just before the perturbation their summary wave is in phase with wave $W_{B}$, because the amplitude of $W_{B A}$ is larger than that of $W_{A}$. Thus the beating now starts with destructive interference of the $\left\{W_{A}+W_{B A}\right\}$ and $W_{B}$ waves when their relative phase is changed due to the perturbation.

In these considerations, the essential feature is the different role of the phase modification when target $A$ starts to be moved. For the sequence $A B$, only wave $W_{A}$ becomes directly phase modulated, whereas wave $W_{A B}$, generated in response, i.e., in an indirect way, is only slightly modulated [see Eq. (4) and $W_{A B}$ in Fig. 6]. This is why the interference of $W_{A}$ and $\left\{W_{B}+W_{A B}\right\}$ has to be considered. For the opposite sequence $B A$ where the downstream target $A$ is moved, waves $W_{A}$ and $W_{B A}$ become directly phase modulated [see Eq. (6) and $W_{B A}$ in Fig. 6]. Thus now the interference of $\left\{W_{A}+W_{B A}\right\}$ and $W_{B}$ has to be considered.

\section{Early perturbation}

We turn now to the case where the polariton starts to be perturbed soon after its creation, at 17 ns. The bottom panel of Fig. 7 shows the velocity profile. The radiative coupling interrupted at the early stage yields the waves $W_{A B}\left(W_{B A}\right)$ to be less developed in amplitude; compare the relevant panels in the Figs. 6 and 7. Moreover, the wave amplitude starts to decay already at about $20 \mathrm{~ns}$. When the radiative coupling of the targets is restored after $\sim 60$ ns the amplitudes $W_{A B}\left(W_{B A}\right)$ start rising again. The perturbation of the radia- tive coupling has two main consequences. At reduced coupling mainly the two scattering paths, represented by the $W_{A}$ and $W_{B}$ waves, interfere. Since the frequency difference between the waves is running within the time interval 20-60 ns their interference yields the amplitude modulation of the wave $\left\{W_{A}+W_{B}+W_{A B}\left(W_{B A}\right)\right\}$ and the relevant beating of the forward scattering intensity in this interval; see the two upper panels of Fig. 7. In addition, the transition to the second scattering stage of the whole system is shifted from $\sim 70 \mathrm{~ns}$ in the case of the unperturbed polariton (see Fig. 4) to nearly $100 \mathrm{~ns}$ when the perturbation is applied. This is because breaking the radiative coupling reduces the effective thickness of the scattering system.

If we compare the scattering patterns for the direct and inverted sequences of the targets we notice that they differ much less drastically as compared to the previous case of late perturbation: the quantum beats are in phase for both sequences, but the beats are less pronounced for the inverted sequence of the targets. This is because upon decoupling in the sequence $A B$ the fields $W_{A}$ and $\left\{W_{B}+W_{A B}\right\}$ are of comparable amplitude, whereas in the sequence $B A$ the amplitudes of the fields $W_{B}$ and $\left\{W_{A}+W_{B A}\right\}$ differ essentially. In addition, the intensity at late times is higher for the $A B$ sequence than for the $B A$ sequence. This comes from two reasons. First, after onset of coupling, in the sequence $A B$ the driving field $W_{A}$ is present at all times, whereas in the sequence $B A$ the driving field $W_{B}$ crosses zero. Second, the response of the downstream $\operatorname{target} B$ at the onset of coupling 

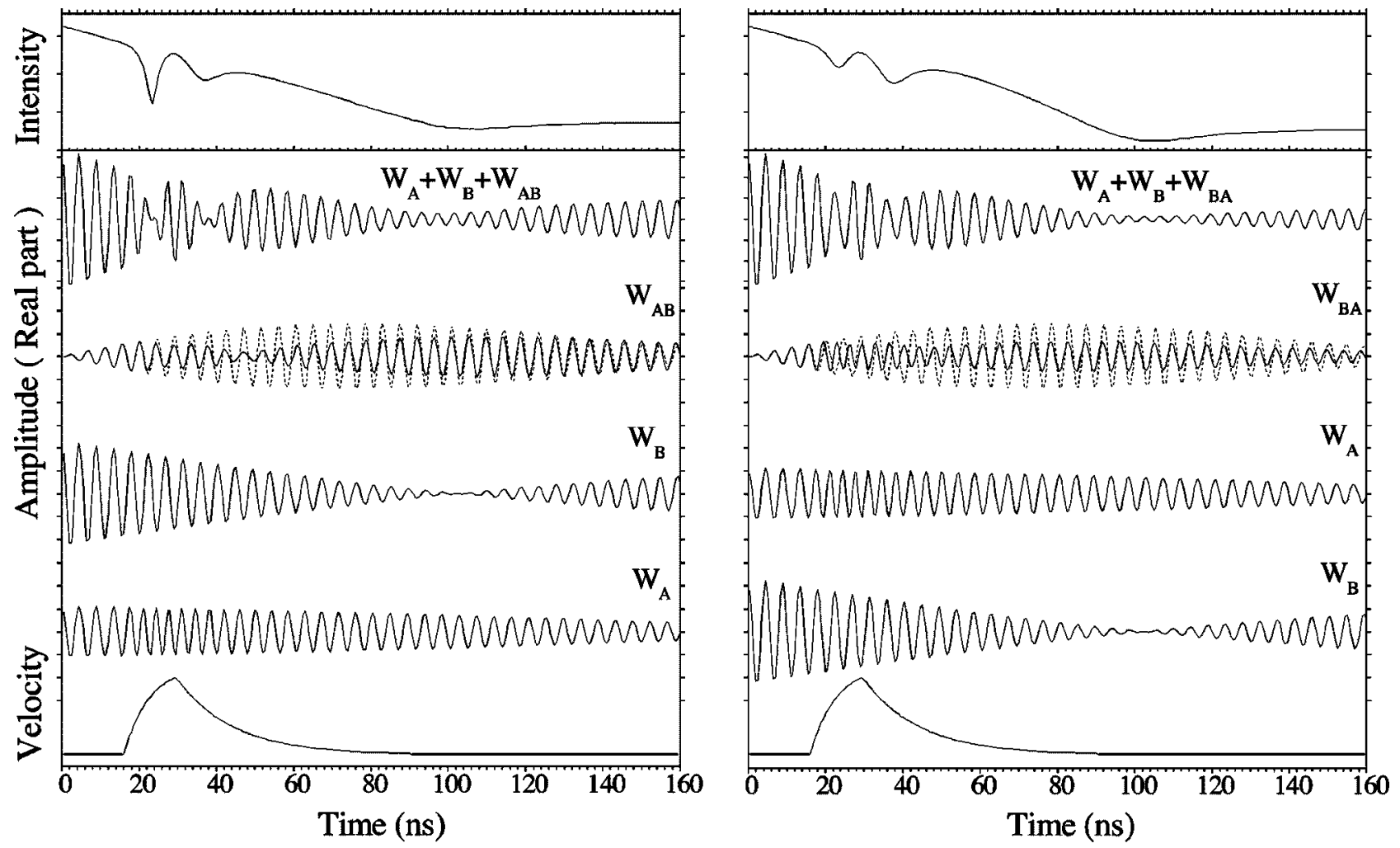

FIG. 7. Effect of inversion at early perturbation (see Fig. 5). The left (right) panel corresponds to the direct (inverted) target sequence $A B(B A)$, respectively. The velocity of target $A$ is depicted by the solid line in the bottom panel. Early perturbation leads to a distinct modification of the overall shape of the system response (see text). However, inversion yields less dramatic consequences as compared to the late perturbation: quantum beats are less pronounced upon inversion. The probability waves $W_{A B}$ and $W_{B A}$ in the absence of perturbation are displayed by dashed lines.

is faster than that of the downstream target $A$ due to its larger thickness [16].

\section{B. Inversion of targets of different thicknesses and different resonance widths}

In these studies the targets having thicknesses of $\sim 1.2$ and $\sim 2.5 \mu \mathrm{m}$ were employed as the parts $A$ and $B$ of the scattering system, respectively. Target $B$ was cooled down to 4.2 K, increasing its Mössbauer thickness by $\sim 28 \%$. In addition, at $4.2 \mathrm{~K}$ the resonance line was broadened to $\sim 10 \Gamma_{0}$ due to magnetic relaxation processes in the material (for details see [10]). A preliminary report on this experiment has been given in [17].

The broadened resonance in target $B$ provides quite different time characteristics for its coherent response. Because of the resonance broadening the target reacts much faster to temporal changes of the external excitation, in particular, to the onset and to the offset of the excitation. A fast response to the onset of the excitation can be explained by the dynamics of $\gamma$-ray scattering in the energy region around the resonance: slow scattering on resonance and fast scattering off resonance [18]. Regarding the offset, the spontaneous emission into the coherent channel proceeds very fast from a target with a broadened resonance. The reason for this acceleration is the fast dephasing of the spectral components due to the broadening of the emitted radiation spectrum, de- scribed by the parameter $q$ [see Eq. (1)] [8]. As discussed below, these features of the response of target $B$ are essential for the polariton propagation through the two-target system.

The general features of the polariton propagation through the unperturbed system are seen in Fig. 4(b). In contrast to the previous case [illustrated in Fig. 4(a)], where both targets are kept at room temperature, the overall shape of the time dependences is changed; in particular, the second scattering stage seems to be largely suppressed. However, in the course of scattering two regimes of polariton propagation can clearly be distinguished. They are characterized by the fast and slow decays of the coherent signal in the time windows 0-50 and 60-160 ns, respectively, revealing a large difference in the responses of the constituent targets. Vanishing intensity at late times is a rather unique situation. It could only be reached because of the particular choice of the thickness of target $B$. As will be shown below the coherent scattering at late times is determined by the sum of two contributions $\left\{W_{A}+W_{A B}\left(W_{B A}\right)\right\}$. For fixed linewidths of the resonances in targets $A$ and $B$ the amplitudes of $W_{A}$ and $W_{A B}\left(W_{B A}\right)$ only depend on the target thicknesses [see Eqs. (1) and (2)]. In order for $W_{A B}\left(W_{B A}\right)$ to cancel $W_{A}$ only the thickness of target $B$ has to be chosen properly. To a large extent this choice is independent of the thickness of target $A$ since $W_{A}$ and $W_{A B}\left(W_{B A}\right)$ depend in a highly similar way on the thickness of target $A$. 

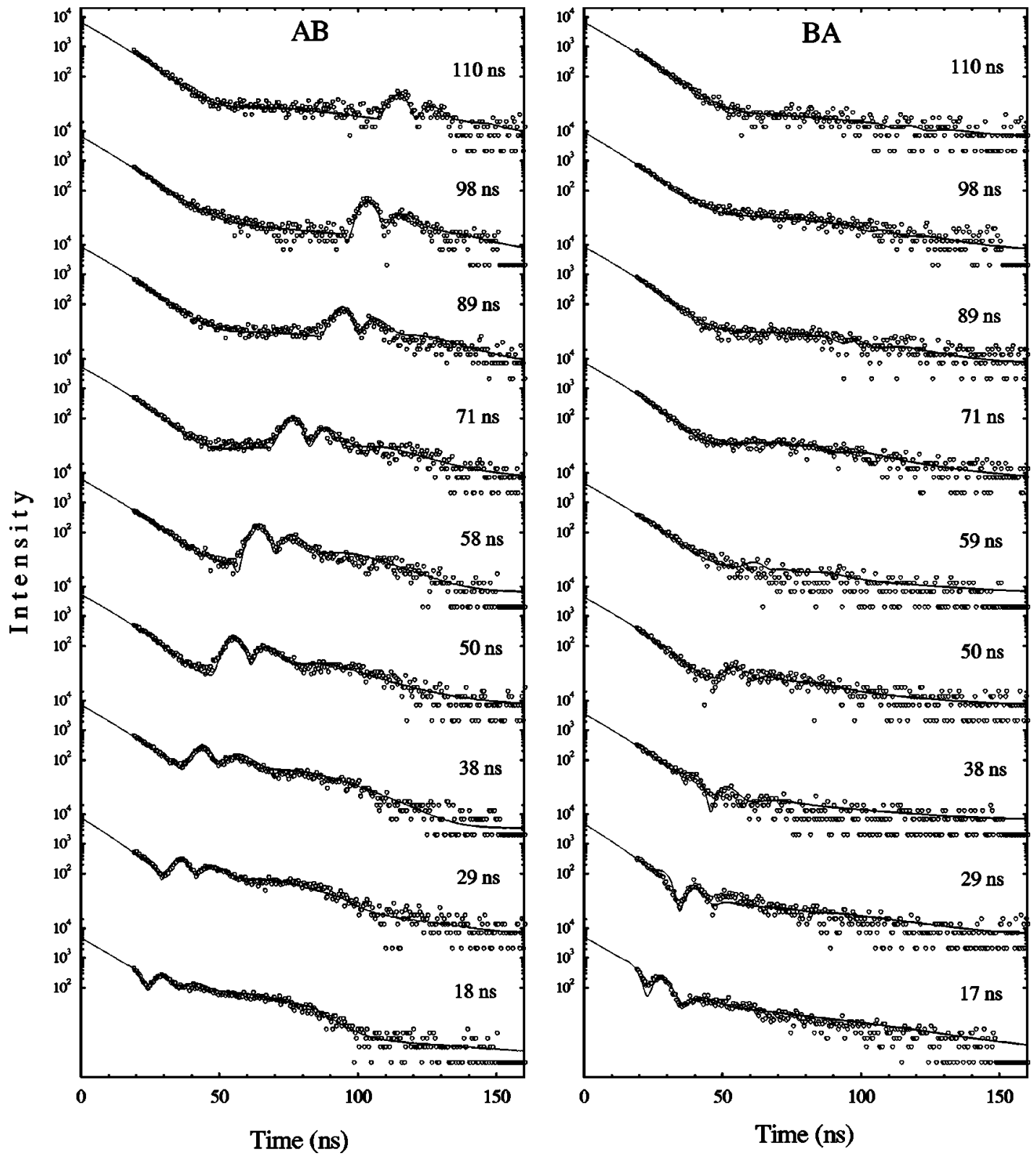

FIG. 8. Experimental time dependences of nuclear forward scattering of synchrotron radiation from a system of two targets $A$ and $B$ manufactured of stainless-steel foils having thicknesses $\sim 1.2 \mu \mathrm{m}\left(T_{A} \approx 10.6\right)$ and $\sim 2.5 \mu \mathrm{m}$, respectively. Target $B$ was cooled to $4.2 \mathrm{~K}$ resulting in a Mössbauer thickness of $T_{B} \approx 28.2$; in addition, at $4.2 \mathrm{~K}$ the resonance line in target $B$ was broadened to $\sim 10 \Gamma_{0}$. The scattering in the system is perturbed by a sudden motion of target $A$ within $\sim 50 \mathrm{~ns}$. The motion starts at different times $t_{01}$ from 17 to 110 ns, as indicated, perturbing the scattering in characteristic stages of its development. The results of the measurements for the direct $A B$ and inverse $B A$ sequences of the targets are presented at the left- and right-hand sides, respectively. Solid lines are computer simulations based on Eqs. (4) and (6).

Figure 8 shows a collection of measured time dependences of the nuclear forward scattering of SR pulses by the system in the presence of perturbation: for different perturbation times and for the two target sequences. The quantum beats caused by the perturbation are well formed in the whole set of dependences obtained for the direct $A B$ sequence of the targets (see left-hand panels). The beating starts from destructive interference for the early perturbations (starting at 18 and $29 \mathrm{~ns}$ ), and then transforms to constructive interference for perturbations at later times.
The time dependences for the inverted $B A$ order of the targets are essentially different. When the start of perturbation crosses the range of $\sim 50 \mathrm{~ns}$ quantum beats are no longer formed. These drastic effects of the inversion of targets we will again analyze separately for the late and early perturbation times.

\section{Late perturbation}

The time dependence where the perturbation started at 98 $\mathrm{ns}$ is a representative example of this case. The wave packets 

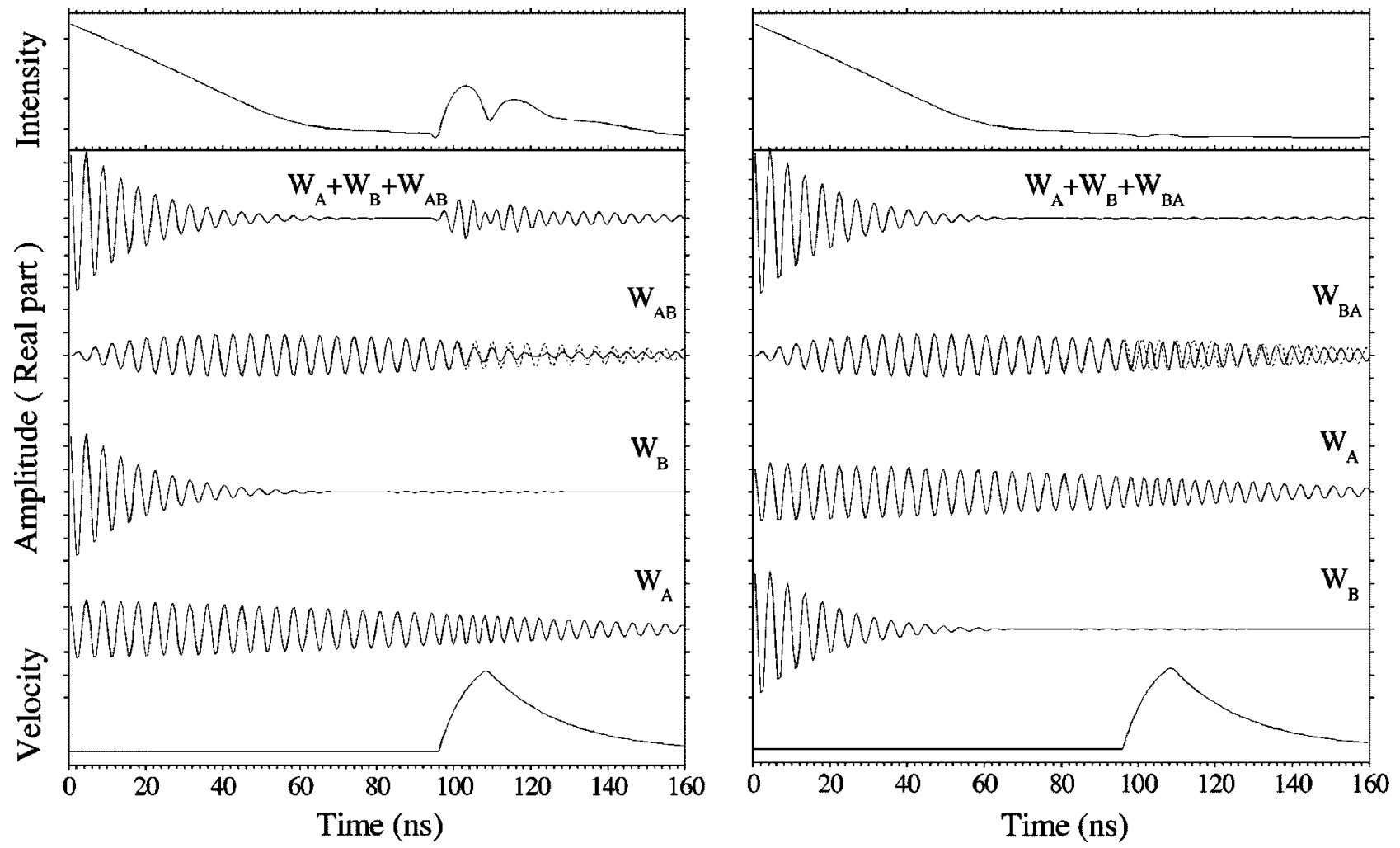

FIG. 9. Effect of inversion at late perturbation (see Fig. 8). The left (right) panel corresponds to the direct (inverted) target sequence $A B(B A)$, respectively. The velocity of $\operatorname{target} A$ is depicted by solid lines in the bottom panel. The pronounced quantum beats observed in the direct sequence of the targets disappear completely after inversion (see the uppermost panels and text). The probability waves $W_{A B}$ and $W_{B A}$ in the absence of perturbation are displayed by dashed lines.

presenting the three scattering paths and the summary wave packet are shown in Fig. 9, along with the forward scattering intensity. The response of target $A$ alone is the same as discussed earlier. Within the time interval $98-160$ ns the wave packet is phase modulated due to the target motion. The response of target $B$ alone is strongly different from that of $\operatorname{target} A$. While target $A$ radiates for the whole time window, target $B$ stops emitting already at about $60 \mathrm{~ns}$ after the excitation by the SR flash. The main reason for this strongly accelerated decay of the wave amplitude $W_{B}$ is the fast dephasing of the spectral components of the forward scattered radiation, as discussed above.

The stage of the fast decay of the coherent signal (see top panel) is determined to a large extent just by the $W_{B}$ scattering path. Within the first $\sim 20 \mathrm{~ns}$ the main contribution comes from constructive interference of the $W_{A}$ and $W_{B}$ waves, where the $W_{B}$ wave plays the dominant role. After this time the increasing amplitude of the $W_{A B}$ wave starts canceling the contribution of the $W_{A}$ wave, so that the whole scattering pattern within the time interval 0-60 ns is formed mainly by the $W_{B}$ channel.

The nuclear double scattering in the composite system runs here only through the $W_{A B}\left(W_{B A}\right)$ scattering channels. In the case under study these channels are less strong than in the previous experiment (see Fig. 6), because of the essentially different resonance widths of the targets. Nevertheless, the targets stay radiatively coupled to a certain degree for most of the time until the motion starts. As a result of our choice of the thickness of target $B$ the amplitude of the $W_{A B}$ wave reaches a value comparable to that of the $W_{A}$ wave. That is why, in the time range until $98 \mathrm{~ns}$ the $W_{A}$ and $W_{A B}$ waves being in opposite phase mostly cancel each other. However, when the upstream $\operatorname{target} A$ is put in motion they quickly reach constructive interference yielding the pronounced bump of the intensity at the begin of the quantum beat structure (left-hand panel). This does not happen when target $A$ is moved in the downstream position (right-hand side). When the motion starts at $98 \mathrm{~ns}$ the $W_{B A}$ and $W_{A}$ waves are frequency modulated in the same way and stay in opposite phase, all the time canceling each other.

Thus, for the different sequences of the targets, the perturbed system exhibits an essentially different behavior: radiating or not radiating coherently in the late stage of scattering. This result clearly shows that the nuclear polariton propagates through the system in different fashions for the different sequences of targets. For the $A B$ sequence at late times before the start of perturbation the polariton propagates only in the upstream target A. The fact that it propagates through this target is indicated by the presence of the wave field $W_{A}$ behind the target $A$. At the same time, the total field behind target $B$ is strongly suppressed because of destructive interference of the wave $W_{A}$ emitted by the upstream target $A$ and the wave $W_{A B}$ generated in the downstream target $B$ due to the double scattering of the radiation first in $A$ and then in $B$. Since there is no wave field behind target $B$ we conclude that the probability amplitude for a nuclear polariton being present in $\operatorname{target} B$ is vanishing. 

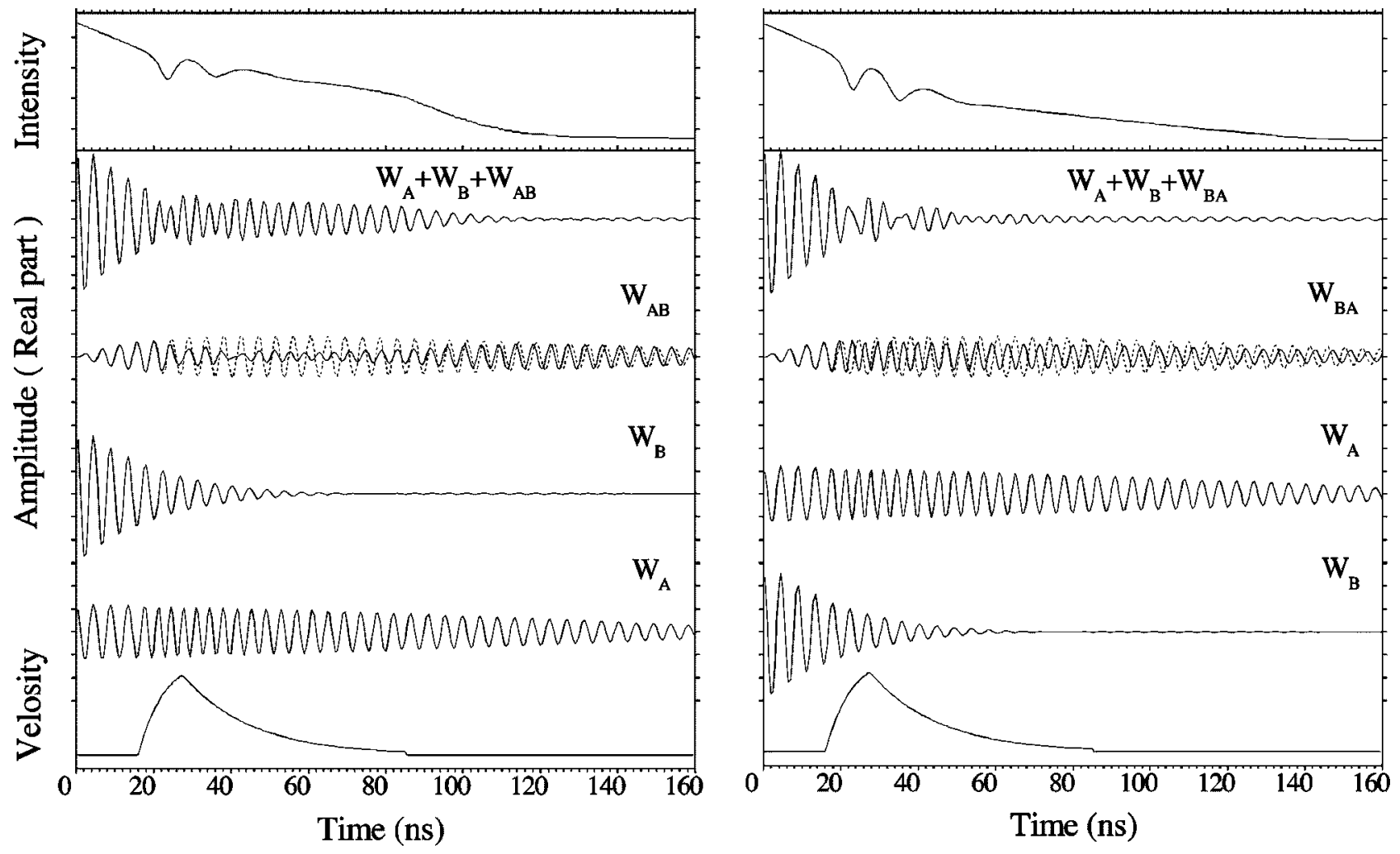

FIG. 10. Effect of inversion at early perturbation (see Fig. 8). The left (right) panel corresponds to the direct (inverted) target sequence $A B(B A)$, respectively. The velocity of target $A$ is depicted by the solid line in the bottom panel. The overall shape of the system response changes considerably when the target sequence is inverted (see text). The probability waves $W_{A B}$ and $W_{B A}$ in the absence of perturbation are displayed by dashed lines.

When the perturbation is applied the interference of $W_{A}$ and $W_{A B}$ results in the quantum beat structure observed (see Fig. 9, left-hand panel). Hence, in the presence of perturbation a polariton can be found in the targets $A$ and $B$. This is indicated by the presence of the wave field $W_{A}$ behind the target $A$ and by the presence of the wave field $\left\{W_{A}+W_{A B}\right\}$ behind the $\operatorname{target} B$. Since the nuclei in $\operatorname{target} B$ are no longer driven by $W_{A}$, the constituent field $W_{A B}$ rapidly decays because of dephasing (similar to $W_{B}$ at early times). Thus also the probability amplitude to find a nuclear polariton in $B$ (related to $W_{A B}$ ) rapidly vanishes with time.

For the $B A$ sequence at late times (in the time window 60-160 ns) there is no polariton in either target. In the upstream target $B$ the polariton vanishes because of the fast decay of the coherent field in this target which is caused by the resonance broadening. In the downstream target $A$ the polariton is strongly suppressed because of destructive interference of the waves $W_{A}$ and $W_{B A}$. In contrast to the former case both waves are generated now in one and the same target: $W_{A}$ by the SR pulse and $W_{B A}$ by the radiation emitted from target $B$. Therefore the phasing of the waves inside the target cannot be modified by perturbation.

Thus in the absence of perturbation the polariton is suppressed in the downstream target for both sequences of targets but in different ways: in the direct sequence the wave $W_{A B}$ is canceled from the outer source (upstream target $A$ ), whereas in the inverted sequence the wave $W_{B A}$ is canceled from the inner source (downstream target $A$ itself). The per- turbation of the radiative coupling of the targets allowed us to distinguish these different modes of polariton suppression.

Even though in the absence of perturbation the polariton vanishes in the downstream target for both sequences, the excitation in this target still remains. However, it is now localized at a particular nucleus, which deexcites via the incoherent channel, i.e., via conversion electrons or $\gamma$ radiation into $4 \pi$ (see Fig. 12 in Ref. [8]). As a consequence, the excitation decays with a time constant corresponding to the natural lifetime. Simulations show that the distributions of the nuclear excitation over the scattering system are strongly dependent on the target sequence [19].

\section{Early perturbation}

This case is illustrated by the time dependence where the perturbation starts at $\sim 17 \mathrm{~ns}$, Fig. 10 . Now the radiative coupling between the targets is interrupted before the excitation of the downstream target by the delayed radiation from the upstream target is saturated.

After decoupling of the targets in their direct sequence the wave $W_{A B}$ decays rather fast because of the rapid response of target $B$ described earlier in this section. Therefore the contribution to the summary wave $\left\{W_{A}+W_{B}+W_{A B}\right\}$ within the time interval 20-90 ns is mainly determined by the $W_{A}$ and $W_{B}$ waves, where the role of the $W_{A}$ wave dominates in the second half of the interval. The intensity between $\sim 40$ and $90 \mathrm{~ns}$ is almost completely determined by the $W_{A}$ scattering path alone. The quantum beats in the direct sequence are 
formed by interference of the waves $W_{A}$ and $\left\{W_{B}+W_{A B}\right\}$.

In the inverted sequence of the targets the wave $W_{B A}$ decays much more slowly. Therefore its contribution to the summary wave during the decoupling period and thereafter turns out to be noticeable until the end of the observation time window. In particular, it is added destructively to the wave $W_{A}$, strongly diminishing the role of the latter. This is the reason for the strong difference in intensity in the time window $\sim 40-90 \mathrm{~ns}$ for the two target sequences. In the inverted sequence, the quantum beats are formed by interference of the waves $W_{B}$ and $\left\{W_{A}+W_{B A}\right\}$.

One should emphasize that the nature of the double scattering represented by $W_{A B}$ and $W_{B A}$ in the time interval $\sim 80-160 \mathrm{~ns}$ is quite different for the direct $A B$ and inverted $B A$ sequences of the targets. In the direct sequence the upstream target $A$ still radiates in the time range after $\sim 80 \mathrm{~ns}$. This means that the downstream $\operatorname{target} B$ is being driven all the time after the motion of target $A$ stops and, hence, the radiative coupling is established again. As a result $W_{A B}$ starts to increase in amplitude acquiring a phase shift of $-\pi$ with respect to $W_{A}$. Therefore, the two scattering channels represented by the probability waves $W_{A}$ and $W_{A B}$ interfere destructively, resulting in a drop of the scattering intensity at $\sim 90 \mathrm{~ns}$ to a very low level. This drop is quite fast due to the accelerated response of target $B$ to the onset of radiative coupling with target $A$. The stepwise fall in intensity is thus a direct fingerprint of the onset of coupling.

In the inverted sequence of the targets the upstream target $B$ no longer radiates in the forward direction after $\sim 60 \mathrm{~ns}$. This means that excitation and deexcitation of the downstream target $A$ by the delayed radiation from the upstream target are separated in time. The energy pumped into target $A$ from target $B$ within the first $\sim 17 \mathrm{~ns}$ is gradually released during all the rest of the time; see the wave $W_{B A}$. Within the time interval between $\sim 60$ and $160 \mathrm{~ns}$, the interference of the two scattering channels presented by the probability waves $W_{A}$ and $W_{B A}$ is now determined by the character of the relative motion of the targets during the perturbation. In the considered example they are only partially phased, yielding a moderate emission from the whole system during this time.

\section{Fitting of the experimental dependences with the theory}

Several parameters describing the time dependences in Eqs. (1), (4), and (6) have to be known to fit the experimental data with the theory. They are the thickness parameters, the parameters of the resonance width, and the velocity parameters. The parameters of the thickness and of the resonance width were obtained by fitting the forward scattering intensity from the individual targets that were employed in the composite scattering system. All these parameters are given in the text above.

The velocity was approximated by the dependence determined below. The perturbation time interval was divided into two parts: $t_{01}$ to $t_{01}+\Delta t$ for the rise and $t_{01}+\Delta t$ to $t_{02}$ for the fall of the velocity. The velocity law within these time intervals was modeled by two exponentials

$$
v(t)=v_{\max }\left[1-e^{-\left(t-t_{01}\right) / \tau_{1}}\right], \quad t_{01}<t<t_{01}+\Delta t,
$$

$$
v(t)=v_{\max } e^{-\left(t-t_{01}-\Delta t\right) / \tau_{2}}\left[1-e^{-\Delta t / \tau_{1}}\right], \quad t_{01}+\Delta t<t<t_{02} .
$$

Time $t_{01}$ denotes the start and $t_{02}$ the stop of motion; $t_{02}$ $\approx t_{01}+60 \mathrm{~ns}$. When fitting the experimental data, four parameters of the velocity profile were varied: $v_{\max }, \Delta t, \tau_{1}$, and $\tau_{2}$. In the experiments where target $B$ was cooled to low temperatures, a constant velocity corresponding to $\sim 1.5 \Gamma_{0}$ originating from the second-order Doppler shift was taken into account. The solid curves on Figs. 5 and 8 present the results of the fitting. Calculations were done with the help of a code based on Eqs. (4) and (6). Slightly different results were obtained for the two kinds of experiments described in Secs. III A and III B. Consistent results for the whole set of dependences displayed in Fig. 5 were reached with the following values of the parameters: $v_{\max } \bumpeq 92 \Gamma_{0}, \Delta t=15 \mathrm{~ns}, \tau_{1}=7.5 \mathrm{~ns}$, and $\tau_{2}=12 \mathrm{~ns}$. Fitting of the data displayed in Fig. 8 yielded the following parameters for the velocity profile: $v_{\max }$ $\triangleq 106 \Gamma_{0}, \Delta t=13 \mathrm{~ns}, \tau_{1}=7.5 \mathrm{~ns}$, and $\tau_{2}=19 \mathrm{~ns}$.

\section{SUMMARY AND CONCLUSION}

The propagation of nuclear exciton polaritons, or simply nuclear polaritons, through a scattering system composed of two spatially separated targets $A$ and $B$ was studied. The targets were highly enriched by resonant nuclei $\left({ }^{57} \mathrm{Fe}\right)$. A nuclear excitonic state was created in both targets almost instantly by a very short flash of synchrotron radiation. The subsequent propagation of nuclear polaritons through the system was investigated by the delayed coherent response of the system in the forward direction.

The theory was restricted by regarding the nuclear resonance in the absence of hyperfine interactions. Within this limitation a general theory of propagation was developed, where the constituent targets were allowed to have an arbitrary resonance energy and different effective resonance thicknesses and resonance widths. In addition, the resonance frequencies of the targets could be modulated in time. Such modulation created a perturbation of the nuclear polariton propagation. The effect of inversion of the targets in the scattering system was the focus of the study.

The response of the system is described in terms of three scattering paths specific for the system. Two paths represent the scattering from the individual targets as if they were alone in the beam and the third path represents the sequential nuclear resonant scattering of radiation in the upstream and downstream targets, where the delayed nuclear radiation from the upstream target is scattered by the nuclei in the downstream target. The scattering paths are described by the probability waves $W_{A}, W_{B}$, and $W_{A B}$, respectively. $W_{A B}$ originates from the radiative coupling of the targets. The mutual amplitude and phase relations of the waves determine the response of the scattering system. A perturbation can influence only the third scattering path, introducing amplitude and phase modulation of the relevant probability wave. The main result of the theory is that in general, $W_{(A B)^{*}} \neq W_{(B A)^{*} \text {, }}$ where asterisks indicate the perturbation of the system, while in the absence of perturbation $W_{A B} \equiv W_{B A}$. Thus the scattering system is commutative in the absence of perturbations 
and is in general not commutative in the presence of a perturbation.

The experimental studies were performed on two kinds of scattering systems. In the system of the first kind the targets differed only by their thicknesses. In the system of the second kind, in addition to different thicknesses the targets also exhibited different resonance widths. The propagation of nuclear polaritons through the system was disturbed by Doppler shifting the resonance energy of one target within a limited time window during the propagation. The inversion of the target sequence strongly affected the polariton propagation in the presence of perturbation. Also the starting time of the perturbation turned out to be essential. The perturbation was most effective when radiative coupling was allowed to develop. Good agreement was found between experimental data and theory. The time dependences of the system response were well fitted with the theory yielding specific parameters of the perturbation, like the perturbation period and parameters defining the velocity profile.

The investigation of nuclear polariton propagation through a two-target system, and, in particular, the question of commutativity of the target sequence is of fundamental interest in the physics of nuclear resonance scattering of synchrotron radiation. It was shown that, in general, the system of two targets is not commutative when the conditions of radiative coupling of the targets are changed during the polariton propagation through the system. A particular case, namely, the Doppler modulation of the radiative coupling, was demonstrated as a model example. However, the radiative coupling of the targets can also be modulated during polariton propagation through the system by other means, causing a dynamical change of the resonance structure in the targets or of the polarization state of the radiation. For instance, this could be an induced relaxation of the hyperfine structure or induced atomic dynamics synchronized with the arrival of the SR pulse, or employment of polarized targets etc.. The rule formulated above, $W_{(A B)^{*}} \neq W_{(B A)^{*}}$, can be applied for the general case of perturbation.

\section{ACKNOWLEDGMENTS}

This work was supported by the Bundesministerium für Bildung, Wissenschaft, Forschung and Technologie under Contracts No. 05 SK8WOA and No. 05 643GUUAI, by INTAS-RFBR under Contract No. 95-0586, and by the RRC Kurchatov Institute. G.V.S. gratefully acknowledges the financing of a stay at the TUM by the Deutsche Forschungsgemeinschaft.
[1] Polaritons, edited by E. Burstein and F. de Martini, Proceedings of the First Taormina Research Conference on the Structure of Matter (Pergamon Press, New York, 1974).

[2] Excitons, edited by E. I. Rashba and M. D. Sturge, Modern Problems in Condensed Matter Sciences Vol. 2 (NorthHolland, Amsterdam, 1982).

[3] G. V. Smirnov, Hyperfine Interact. 27, 203 (1986).

[4] J. Frenkel, Phys. Rev. 37, 17 (1931).

[5] G. T. Trammell, in Chemical Effects of Nuclear Transformations, Prague, 1960 (International Atomic Energy Agency, Vienna, 1961), Vol. 1, p. 75.

[6] A. M. Afanas'ev and Y. Kagan, JETP Lett. 2, 81 (1965).

[7] D. F. Zaretskii and V. V. Lomonosov, Sov. Phys. JETP 21, 243 (1965).

[8] G. V. Smirnov, Hyperfine Interact. 123/124, 31 (1999).

[9] U. van Bürck, Hyperfine Interact. 123/124, 483 (1999).

[10] W. Potzel, U. van Bürck, P. Schindelmann, G. M. Kalvius, G. V. Smirnov, E. Gerdau, Y. V. Shvyd'ko, H. D. Rüter, and O. Leupold, Phys. Rev. A 63, 043810 (2001).

[11] P. Schindelmann et al., Phys. Rev. A 65, 023804 (2002).

[12] Y. Kagan, A. M. Afanas'ev, and V. G. Kohn, J. Phys. C 12,
615 (1979).

[13] G. V. Smirnov, U. van Bürck, J. Arthur, S. L. Popov, A. Q. R. Baron, A. I. Chumakov, S. L. Ruby, W. Potzel, and G. S. Brown, Phys. Rev. Lett. 77, 183 (1996).

[14] G. V. Smirnov and W. Potzel, Hyperfine Interact. 123/124, 633 (1999).

[15] W. Potzel et al., Hyperfine Interact. 151/152, 263 (2003).

[16] G. V. Smirnov, Y. V. Shvyd'ko, O. S. Kolotov, V. A. Pogozhev, M. Kotrbova, S. Kadechkova, and I. Novak, Sov. Phys. JETP 59, 875 (1984).

[17] U. van Bürck, W. Potzel, P. Schindelmann, G. V. Smirnov, S. L. Popov, E. Gerdau, Y. V. Shvyd'ko, H. D. Rüter, and O. Leupold, Hyperfine Interact. 141/142, 151 (2002).

[18] G. V. Smirnov and Y. V. Shvyd'ko, Sov. Phys. JETP 68, 444 (1989).

[19] G. V. Smirnov (unpublished).

[20] Y. V. Shvyd'ko and G. V. Smirnov, J. Phys.: Condens. Matter 1, 10563 (1989).

[21] G. V. Smirnov, Hyperfine Interact. 97/98, 551 (1996).

[22] M. Haas, V. Hizhnyakov, E. Realo, and J. Jōgi, Phys. Status Solidi B 149, 283 (1988). 